Références de la publication: Dagiral E., Peerbaye A. (2010), «La construction et la diffusion de l'information sur les maladies rares ». In Romeyer H. (dir.), La santé dans l'espace public, Presses de l'Ecole des Hautes Etudes en Santé Publique, pp. 149-158.

\title{
La construction et la diffusion de l'information sur les maladies rares
}

\author{
Eric Dagiral et Ashveen Peerbaye
}

\section{Introduction}

Les maladies rares sont des maladies qui affectent moins d'une personne sur 2 000. Parmi les 7000 maladies rares recensées aujourd'hui, 80\% environ ont une origine génétique identifiée. Malgré la faiblesse des cas, le nombre total de personnes atteintes par ces maladies est important (6 à $8 \%$ de la population mondiale ; 3 millions de Français). Il s'agit la plupart du temps d'affections précoces, sévères, chroniques, invalidantes et dégénératives, qui mettent en jeu le pronostic vital. Ces maladies sont marquées parl'insuffisance des connaissances scientifiques à leur sujet, l'absence de traitements efficaces, et de fortes inégalités d'accès aux traitements et aux soins existants. Elles confrontent les individus qu'elles concernent (malades et proches, chercheurs, médecins, professionnels de santé...) à des situations où l'ignorance pèse fortement sur la recherche thérapeutique, la réalisation d'un diagnostic, l'orientation vers des spécialistes, l'organisation des soins, ou encore la prise en charge socio-économique des malades.

Cet article est issu d'une enquête ethnographique en cours, portant sur les modes de coordination des acteurs composant la Plateforme Maladies Rares, entité qui intègre dans un même lieu cinq organisations travaillant sous des rapports spécifiques pour les personnes atteintes de maladies rares ${ }^{1}$. Certaines sont davantage orientées vers la recherche, tandis que d'autres s'adressent plus directement aux malades, que ce soit par le travail de coordination des associations de malades qu'elles réalisent en France ou en Europe, ou par l'écoute et

\footnotetext{
${ }^{1}$ Les résultats présentés ici sont issus d'une recherche collective en cours (2008-2010), menée avec Robin Foot (LATTS) dans le cadre d'un Partenariat Institutions Citoyens (PICRI), financée et soutenue par la Région Ile-deFrance. Les données ont été principalement recueillies à travers des entretiens approfondis et des séances d'observation systématiques sur l'ensemble de la plate-forme, avec une attention particulière apportée aux modes de coordination entre les différents acteurs, et les supports concrets d'interaction et de mobilisation. Pour une présentation de la recherche: http://www.iledefrance.fr/recherche-innovation/picri/projets-2007/maladierare/
} 
l'aide apportées aux malades, à leurs proches et aux professionnels, par le truchement d'un portail web et d'un centre d'appel. L'activité de cette plate-forme est emblématique des évolutions récentes observées en matière de transformations des relations entre professionnels de santé, malades et associations.

Si les associations de malades ont entretenu dès le début du $20^{\mathrm{e}}$, et surtout à partir de la fin de la deuxième Guerre Mondiale, des liens significatifs avec l'appareil de recherche biomédicale et le système de santé ${ }^{2}$, c'est l'irruption de l'épidémie de sida qui a indiscutablement provoqué, au début des années 1990, un intérêt renouvelé pour cette thématique de recherche. Tout un ensemble de travaux s'intéressent alors à la construction du mouvement associatif autour de maladies particulières (principalement le sida et certaines maladies génétiques), et mettent en lumière la manière dont les relations entre acteurs associatifs et professionnels façonnent la définition collective de ces maladies, et participent à la recomposition du système de soins ${ }^{3}$. Ces travaux montrent le rôle important et diversifié des associations, et permettent de mesurer l'impact grandissant de leurs actions, tout en soulignant des innovations par rapport aux modalités d'intervention traditionnelles: l'implication de certaines associations, loin de se cantonner à l'aval des dispositifs de santé et de recherche biomédicale, remonte désormais très loin en amont pour empiéter sur le territoire jusqu'alors indiscuté des spécialistes. Ces associations remettent en question - avec souvent d'importants moyens politiques, financiers, scientifiques et technologiques - le monopole des experts traditionnels sur l'orientation de la recherche et les modalités d'organisation des systèmes de santé... (Rabeharisoa et Callon, 1999; Barbot, 2002; Dodier, 2003 ; Epstein, 2008). L'originalité de ces «groupes concernés émergents » tient cependant moins au fait qu'ils sont composés de profanes ou d'experts inattendus, mais davantage à la manière dont ils parviennent à articuler des enjeux de recherche avec des questions de citoyenneté. Plus encore, ce sont les formes d'organisation et de coordination que ces groupes promeuvent qui apparaît comme trait distinctif : des collectifs hybrides ouverts, auxquels sont conviés les profanes ; la cognition et l'action distribuées; et une forme de coordination flexible, souvent en réseaux. Sous certaines conditions, ces groupes parviennent à imposer de nouvelles articulations entre recherche scientifique et identités politiques, et contribuent à reconfigurer les marchés et les systèmes de politique publique (Callon et Rabeharisoa, 2008).

\footnotetext{
${ }^{2}$ Cette histoire est bien documentée, en particulier pour les cas de la poliomyélite (Benison, 1972) et du cancer (Patterson, 1987 ; Pinell, 1992).

${ }^{3}$ Pour des exemples de ces travaux initiaux, se référer en particulier à Carricaburu, 1993 ; Paterson et Barral, 1994 ; Epstein, 1995.
} 
Nous présentons brièvement les enjeux politiques et informationnels liés à la problématique de la rareté des maladies, avant d'exposer l'agencement du dispositif d'information et de communication mis en place. Notre étude fait ressortir les difficultés à faire émerger un espace public et à organiser la production et la diffusion d'informations dans un contexte marqué par la rareté et l'ignorance sociale des maladies. Elle pointe en particulier l'importance du travail d'articulation entre différentes scènes sociales pour qu'une infrastructure sociotechnique (reposant fortement sur Internet) parvienne à inscrire durablement et efficacement la question des maladies rares dans l'espace public.

\section{Problématiser la rareté des maladies dans l'espace public}

L'émergence des «maladies rares » comme catégorie politique, susceptible d'objectiver un problème de santé publique et de participer à sa mise sur agenda, est relativement récente. La force de cette expression tient probablement à sa capacité à renouveler la problématique de ces maladies, invisibles compte tenu du faible nombre de malades atteints, pris isolément, maladie par maladie. Sans qu'il soit possible d'en retracer ici une généalogie approfondie, notons toutefois que l'opération consistant à regrouper sous le critère de la rareté un ensemble hétérogène de pathologies émerge à la fin des années 1970 aux Etats-Unis, et vers la fin des années 1990 en Europe. Dans les deux cas, la catégorie est le fruit d'un intense travail de mobilisation d'associations de malades et de leurs proches (Huyard, 2007), en interaction avec les pouvoirs publics, qui se déploie parallèlement sur plusieurs fronts.

Un premier front de la mobilisation se cristallise autour de textes législatifs portant sur la régulation d'une catégorie spécifique de médicaments - dits orphelins - définis par la rareté des pathologies ciblées. L'Orphan Drug Act, adopté en 1983 aux Etats-Unis, accorde le statut de médicament orphelin aux médicaments concernant des pathologies affectant moins d'une personne sur 200000 aux Etats-Unis. Ces médicaments bénéficient d'un ensemble d'incitations économiques pour encourager les entreprises pharmaceutiques à développer et commercialiser ce qui serait autrement des produits non-rentables. À la fin des années 1990, une mobilisation européenne se met en place afin d'obtenir le vote d'un règlement européen relatif aux médicaments orphelins équivalent à l'Orphan Drug Act américain. Ce règlement est adopté en 1999, et est opérationnel depuis 2000, sous l'impulsion décisive de l'European 
Organization for Rare Diseases (Eurordis), association créée en 1997 avec cet objectif explicite, et regroupant initialement l'Association Française contre les Myopathies (AFM), AIDES, la Ligue nationale contre le Cancer et Vaincre la Mucoviscidose.

Un deuxième front de mobilisation est organisé autour de la recherche scientifique sur les maladies rares. Au début des années 1990, des travaux sont entamés afin de caractériser les maladies génétiques ( $80 \%$ des maladies rares) et comprendre les mécanismes du génome humain. L'AFM va jouer dans ce domaine le rôle d'un quasi-acteur public en matière de financement et de stimulation de la recherche scientifique, dans un domaine que la recherche académique et industrielle n'avait jusqu'alors pas beaucoup investi (Rabeharisoa et Callon, 1995 ; Peerbaye, 2004). Progressivement, à la focale presque exclusivement génétique utilisée pour aborder ces maladies se substitue une problématisation en termes de rareté, au fur et à mesure que les recherches se développent non seulement sur le plan « fondamental» (biologie moléculaire, génomique) mais aussi en recherche clinique et en essais thérapeutiques. Le rapport présenté en 2001 au Conseil économique et social par Bernard Barataud, alors président de l'AFM, intitulé Cinq mille maladies rares, le choc de la génétique (Barataud, 2001) illustre fort bien ce mouvement.

Un troisième front de mobilisation est structuré autour de ce que se caractérise comme un travail de lutte contre l'ignorance médico-sociale dont sont victimes les maladies rares. Ce travail passe en particulier par des efforts coordonnés de la part des acteurs pour améliorer l'accès au diagnostic et ainsi lutter contre l'errance diagnostique ; pour informer les malades et leurs proches sur les aides auxquels ils ont droit (prise en charge des soins, aides financières, techniques et humaines); pour organiser des parcours de soins efficaces et y orienter les malades. De manière symétrique, il passe par des efforts pour informer médecins, professionnels de santé et administrations publiques sur ce «public » particulier que forment les malades rares. Sur ce front, également, la stratégie d'une problématisation autour de la rareté a émergé, consistant à subsumer l'hétérogénéité des maladies sous la communauté des multiples difficultés que rencontrent les personnes affectées.

Le concept de « rareté » permet donc de pallier trois types de défaillance qui constituaient autant de trappes où «disparaissaient » les malades. La première concerne la défaillance du marché à réguler l'offre de médicaments pour des marchés réduits et non-rentables, sur lesquels les firmes pharmaceutiques ne pouvaient attendre un retour sur investissement. Cette 
défaillance du marché a «produit » les médicaments orphelins, c'est-à-dire des médicaments pour lesquels des investissements en recherche et développement ne sont envisageables que si une forme d'intervention publique (aides financières, dispositions spécifiques concernant par exemple l'autorisation de mise sur le marché) est assurée. La deuxième défaillance est d'ordre cognitif. La rareté des cas rend problématique l'identification des maladies par les médecins car le corps médical est dans l'incapacité d'accumuler et de distribuer une compétence sur ces maladies. La troisième, enfin, porte sur une défaillance du système de santé publique, nécessitant une reconfiguration de ses dispositifs traditionnels afin de faire face à des maladies longtemps jugées trop spécifiques pour pouvoir être prises en compte. L'errance diagnostique est le corollaire de cette situation, le désintérêt des acteurs pour ces maladies et la difficulté à les appréhender et les représenter se renforçant mutuellement.

La catégorie «maladies rares », on le voit, a été élaborée au sein d'un collectif hétérogène et à travers des dispositifs réglementaires spécifiques, pour devenir en l'espace d'une vingtaine d'années un « objet frontière », visant à inscrire les enjeux des maladies rares dans le régime de la médecine industrielle (Huyard, 2009). Cependant, si la catégorie a réussi à émerger en même temps qu'elle dessinait un nouvel espace de mobilisation et d'activités de recherche, son inscription durable et efficace dans l'espace public requiert également l'invention d'un dispositif apte à articuler deux dimensions qui peuvent sembler irréconciliables : pallier un déficit de connaissances fiables et spécialisées, tout en diffusant des représentations générales à destination d'un public indéterminé.

\section{Une plate-forme pour les maladies rares : produire une information fiable et la publiciser}

En 2001, le Ministre délégué à la santé inaugure, dans les locaux de l'hôpital Broussais (à Paris, dans le $14^{\mathrm{e}}$ arrondissement), la Plateforme Maladies Rares. Cette plateforme, créée sous l'impulsion de l'AFM (qui la finance en grande partie), rassemble les principaux acteurs mobilisés en faveur des personnes atteintes de maladies rares et de leurs familles, sous la forme de deux réseaux associatifs et de trois structures professionnelles :

- l'Alliance Maladies Rares, fédération nationale de plus de 170 associations de malades ; 
- Eurordis, réseau européen représentant plus de 280 associations de maladies rares sur une trentaine de pays ;

- Orphanet, portail Internet européen d'information sur les maladies rares et les médicaments orphelins, hébergé et mis en œuvre par un service commun de l'Inserm ;

- Maladies Rares Info Services (MRIS), service téléphonique d'écoute, d'information et d'orientation à destination des personnes concernées par les maladies rares ;

- et un Groupement d'Intérêt Scientifique, le GIS Institut des Maladies Rares, qui coordonne et développe la recherche scientifique sur les maladies rares.

Regroupées au sein d'une plate-forme commune, les cinq entités n'en conservent pas moins leur identité et leur autonomie organisationnelles. Luttant toutes contre les maladies rares, elles continuent cependant de poursuivre des missions propres à chaque entité dans ce cadre. Mutualisant des moyens techniques, humains et logistiques, et disposant d'un budget annuel commun, elles assurent chacune séparément la recherche de leurs financements. Cette formule organisationnelle à la fois souple et complexe (qui n'est pas sans poser toutefois quelques problèmes aux acteurs eux-mêmes) a incontestablement favorisé, aux dires des intéressés, la mise en place de collaborations entre les entités. Elle a permis également d'accroître la visibilité et la légitimité, au sein des politiques publiques de santé, de ce «milieu» des maladies rares en émergence. Ainsi, en l'espace de moins de dix ans en France, la mobilisation qui donne corps aux «maladies rares », en tant que catégorie pour penser un problème de santé publique, obtient leur inscription à l'agenda comme l'une des cinq grandes priorités de la loi relative à la politique de santé publique du 9 août 2004. Le «Plan national maladies rares 2005-2008 », lancé par les ministères en charge de la Santé et de la Recherche, vient donner corps à cet engagement.

Parmi les «dix axes stratégiques» retenus par ce Plan (sous-titré «Assurer l'équité pour l'accès au diagnostic, au traitement et à la prise en charge ») ${ }^{4}$, la dimension « informationnelle » est particulièrement mise en avant, tant pour les malades et leurs proches, que pour les professionnels de santé et le grand public. Cette dimension se retrouve dans la quasi-totalité des axes, mais apparaît le plus explicitement dans l'axe 3 du Plan: «Développer l'information pour les malades, les professionnels de santé et le grand public concernant les maladies rares ». Cet axe est piloté par la Direction générale de la Santé (DGS),

\footnotetext{
${ }^{4}$ Le texte du Plan est disponible sur le site du Ministère de la santé : http://www.sante.gouv.fr/htm/actu/maladie_rare/plan.pdf
} 
en coordination avec l'Institut National de Prévention et d'Education (INPES), l'Institut national de la santé et de la recherche médicale (Inserm) et les associations de malades. Presque tous les acteurs participant à la réalisation de cette mission de production et de communication de l'information sur les maladies rares se trouvent sur la plate-forme ${ }^{5}$. Deux entités sont concernées au premier chef : le portail internet Orphanet $^{6}$, qui repose notamment sur une base de données en ligne, et le centre d'appel téléphonique du MRIS. Mais au-delà de ces deux services dédiés à l'information, l'ensemble des acteurs concourt à la co-production de l'information, qu'il s'agit avant tout en amont d'élaborer, en identifiant, définissant et classifiant des maladies.

Le site d'Orphanet, qui existe depuis 1997 indique sur sa page d'accueil avoir pour mission d'«améliorer la qualité des soins médicaux pour les Maladies Rares » et "fournir des services adaptés à la communauté des maladies rares» auprès de quatre types de publics: professionnels, patients, associations et industriels. Le portail est à visée d'information médicale à destination du grand public comme des professionnels de santé (plusieurs niveaux : articles scientifiques, bases de données, résumés, fiches grand public...), et couvre 2500 maladies rares sur les 6000 environ référencées. Chaque maladie est caractérisée et classifiée ${ }^{7}$, accompagnée d'un résumé daté la décrivant, et assortie d'une liste de signes cliniques.

Le MRIS, lui, entend "écouter, informer, orienter » les malades ou proches de malades, et dans une moindre mesure, les professionnels de santé. Bien que son histoire soit nettement antérieure au Plan National Maladies Rares, ses missions sont réaffirmées avec force dans le $3^{\text {ème }}$ axe dudit plan :

«Depuis quelques années, les campagnes médiatiques développées par les associations et notamment le "Téléthon » et les «Opérations nez rouge » ont sensibilisé la société française aux maladies rares ou orphelines. Toutefois, les malades et leurs familles rencontrent des difficultés majeures pour s'informer et s'orienter dans le système de soins, ce qui entraîne une errance diagnostique qui altère la qualité de la prise en charge et accrô̂t leur sentiment d'isolement » (p. 13)

Conjointement au portail référent d'informations en ligne, l'information par téléphone répond à plusieurs objectifs complémentaires. Premièrement, elle vise à tempérer la fracture numérique - tout en répondant également aux courriers électroniques (qui représentent un peu

\footnotetext{
${ }^{5}$ A l'exception de la Fédération des Maladies Orphelines, qui dispose d'un service d'écoute téléphonique, mais ne fait pas partie de la plate-forme.

${ }^{6} \mathrm{http}: / /$ www.orpha.net

${ }^{7}$ Par les critères suivants : un numéro Orphanet, des synonymes, sa prévalence (exceptionnelle, 1-9/100 000...), son hérédité et âge d'apparition, des codes correspondants à d'autres classifications (CIM10, MIM).
} 
plus de 20\% du volume des «appels» depuis 2007). L’information sur la santé ne peut en effet pas relever exclusivement de la consultation d'Internet. La ligne téléphonique permet d'articuler information et écoute, afin de faire émerger une demande d'information, qui n'est pas nécessairement formulée dans les situations d'errance diagnostique (environ $25 \%$ des appels $^{8}$ ). Enfin, si l'information téléphonique ne doit jamais déboucher sur un diagnostic, domaine réservé du médecin, les écoutantes doivent s'assurer que l'orientation vers un spécialiste de référence est en cours, et si tel est le cas, que la prise en charge sociale éventuelle est effective. Au cours des appels, elles s'assurent donc progressivement que l'ensemble de ces domaines d'action est couvert et interrogé, et l'orientent donc selon la situation.

Le cadre général dans lequel les pouvoirs publics élaborent habituellement des dispositifs d'information concernant une maladie particulière ne peut être transposé aisément dans le cas des maladies rares. En effet, lorsque dans le paysage français de la «téléphonie santé », les numéros d'appel se sont multipliés, cela s'est presque toujours accompli en liaison avec une pathologie ou un comportement particuliers (sida, tabagisme, alcoolisme, etc.) Dans ce contexte, le projet d'une ligne téléphonique de santé dédiée aux maladies rares est apparu pertinent dès les années 1990, avec la création par l'AFM, en 1995, du service « Allo-Gènes », soutenu par le ministère de la Santé. Cependant, les maladies sur lesquelles informer les appelants sont extrêmement nombreuses - entre 6000 et 8000 selon les dénombrements réalisés. Comment, dans ces conditions, former le personnel en charge de délivrer une information aussi spécialisée, variée et sensible ? Comment informer efficacement dans le cadre d'une interaction téléphonique délicate, dont l'enjeu est souvent de contribuer à faire émerger une demande ? Comment, enfin, informer lorsque l'appel provient d'une personne en situation d'errance diagnostique, et qui s'oriente in fine vers ce service, interpellée par l'expression «maladies rares »? Autrement dit, comment renseigner lorsqu'une maladie n'est pas identifiée avec certitude, voire pas identifiée du tout? Un tel service, en se présentant comme dédié aux «maladies rares », concerne et capte un public particulièrement atomisé, ce qui complique considérablement la dissémination de l'information.

Si Internet a pu figurer, au milieu des années 1990, l'opportunité majeure pour l'information de santé sur les maladies rares, un serveur d'information ne suffit pas à lui seul à rendre

\footnotetext{
${ }^{8}$ Le reste des appels est le fait de personnes à la recherche d'une information liée à une maladie diagnostiquée.
} 
visibles ces informations : il n'en assure pas la promotion, et reste peu représenté dans l'espace public médiatique, davantage investi par les associations de malades. Ceci explique que la construction collaborative, qui s'effectue en réseaux et repose sur une circulation de l'information articulée autour du serveur d'information Orphanet, ne peut pas s'y réduire pas exclusivement.

Le fait que les maladies concernées par cette politique soient nombreuses et rares entraîne en effet une série de conséquences qui impactent directement la mise en place d'un dispositif d'information de santé, tant au niveau de la définition de l'information elle-même, que de ses modes de production et de communication. Le premier enjeu en la matière, considérable, consiste à construire une information scientifique fiable sur des maladies progressivement identifiées, mais peu ou mal connues. Ce travail de sélection, de définition et d'appariement des informations autour des maladies est coordonné et assuré principalement par Orphanet, et s'appuie sur les travaux de recherches de spécialistes à travers le monde. L'enjeu consiste à tirer parti des possibilités offertes par Internet pour constituer un serveur d'informations de référence, en profitant de l'affaiblissement des coûts de diffusion, de l'internationalisation des coopérations et des possibilités de mise à jour décisives pour ce type de projet.

À côté de ces avantages déterminants, l'hétérogénéité des informations disponibles en ligne et le succès des contributions et discussions non professionnelles en matière de santé installent durablement la question de la fiabilité des informations dans l'espace public en ligne. Si les usagers des forums n'ont pas nécessairement vocation à être prescripteurs en matière de médicaments, ces espaces de discussion canalisent une partie des controverses liées à la fiabilité et à la signature de l'information (Adams et Bal, 2009). Depuis la figure du patientexpert (Fox, Ward, O'Rourke, 2005) jusqu'aux reconfigurations de la présentation de la parole médicale sur internet (Hardey, 1999), nombreux sont les auteurs qui identifient dans ces pratiques rendues aisément accessibles de nouveaux questionnements posés aux acteurs professionnels (Akrich et Méadel, 2002).

L'information sur les maladies rares pose donc, au moins, un double problème. Le premier, qui unit les chercheurs, les associations de malades et les institutions de santé, est la rareté de l'information: le déficit de savoir est au départ de la mobilisation. Sans une meilleure appréhension des maladies, aucune perspective de soin et de traitement n'est, à terme, possible. Ainsi l'enjeu majeur est-il de recenser les maladies rares afin de pouvoir mener des recherches à leur sujet, de créer de la connaissance, et de traduire cette connaissance en 
information à destination de différents publics. C'est, on l'a vu, le rôle essentiel dévolu à Orphanet et à l'Inserm (en relation avec le GIS Maladies Rares), depuis la reconnaissance et l'ajout d'une maladie au sein de la classification des maladies rares, jusqu'à la rédaction d'une information comprenant plusieurs niveaux de sophistication. Le second enjeu, relève de la jeunesse du concept de maladies rares, de la «rareté » des personnes concernées, et plus encore de leur situation d'isolement et de l'hétérogénéité considérable de leurs maladies. C'est cette situation qui a conduit à mettre en place un dispositif d'information qui vise à sensibiliser à l'existence et à la problématique des maladies rares, à informer sur ces milliers de maladies, et à articuler ses différents publics (malades, proches de malades, associations de malades, médecins, chercheurs, acteurs de l'industrie pharmaceutique, du monde politique) pour rendre possible l'émergence d'un milieu de recherche et de soin adapté, et plus encore, aider à le pérenniser. Dans ce cas, la construction et la diffusion de l'information sont indissociablement des outils d'information et de communication, par et pour la plate-forme maladies rares.

Dans ce contexte, la production même de cette information, et sa traduction à destination de publics différents (malades, proches, médecins, chercheurs, etc.), sont au cœur des enjeux de la Plateforme Maladies Rares. L'activité téléphonique ne serait pas possible sans la consultation d'une base de données relationnelle agençant l'information disponible sur ces maladies. C'est dans cette perspective que l'intégration des technologies d'information et de communication que nous avons observée prend tout son sens: l'activité d'écoute et d'information entre en synergie avec la consultation d'une information extrêmement spécialisée, et invite à prendre en compte de manière conjointe la construction de l'information, de ses outils de diffusion et de médiatisation (Boczkowski et Lievrouw, 2008).

\section{Conclusion}

L'articulation d'un serveur d'information web et d'un service d'information téléphonique répond, en premier lieu, aux attentes et besoins des acteurs concernés, qu'ils soient malades, médecins, chercheurs, etc. En second lieu, ce dispositif informationnel compose l'infrastructure qui permet le déploiement et la circulation d'un corpus d'informations reconnues et mobilisables par le système de santé, comme en témoigne le travail en cours de révision de la classification internationale des maladies en vue d'y inclure des maladies rares. Cette dimension infrastructurelle, pour autant, pose problème lorsqu'on s'intéresse aux 
reconfigurations de l'espace public en matière de santé. L'activité de refonte d'une nomenclature ou les mises à jour régulières d'une vaste base de données relationnelle restent des actions peu observées, voire invisibles ${ }^{9}-$ de par leur complexité notamment - dans les discussions généralistes sur la santé. Le dispositif ne se suffit donc pas à lui-même pour assurer sa visibilité, malgré la simplicité et/ou la gratuité de l'accès sur Internet ou par téléphone. C'est donc hors de ces espaces d'interaction et de communication que l'affirmation de la nécessité d'un dispositif original de lutte pour les malades se prolonge, impulsé notamment par les collectifs d'associations de malades et quelques campagnes institutionnelles. Les médias dits de masse, télévision et radio particulièrement, sont la cible de ces campagnes de communication et d'information. Ainsi l'évènement médiatique majeur pour la mise en visibilité publique des maladies rares reste encore le Téléthon ${ }^{10}$ organisé par l'AFM avec France Télévisions, et dont les éditions récentes ont été marquées par l'utilisation généralisée de la catégorie de «maladies rares ${ }^{11}$.

\section{Références bibliographiques}

Adams S., Bal R. (2009), "Reliability Reconstructing Traditional Boundaries in the Gray Areas of Health Information Review on the Web", Science, Technology, \& Human Values, 34(1), pp. 34-54.

Akrich M., Méadel C. (2002), «Prendre ses médicaments/prendre la parole : les usages des médicaments par les patients dans les listes de discussion électroniques», Sciences Sociales \& Santé, 20, (1), p.89-116.

Barbot J. (2002), Les malades en mouvements. La médecine et la science à l'épreuve du sida, Paris, Balland.

Benison S. (1972), The history of polio research in the United States : appraisal and lessons. In Holton G., The Twentieth-century sciences: Studies in the biography of ideas. New York, W. Nortob.

\footnotetext{
${ }^{9}$ Sur l'invisibilité sociale des infrastructures de type bases de données dans de nombreux contextes, cf. Bowker, 2005.

${ }^{10}$ Sur le traitement médiatique du Téléthon, voir Cardon et al., 1999.

${ }^{11}$ Si le Téléthon constitue l'événement majeur, au sein duquel se déroule une "marche des maladies rares » particulièrement mise en avant et médiatisée, un programme télévisé tel que «Le magazine de la santé » (France 5) a été également à plusieurs reprises l'occasion d'une intervention de la médecin-généticienne de l'équipe du MRIS.
} 
Boszkowski P., Lievrouw A. (2008), "Bridging STS and Communication Studies: Scholarship on Media and Information Technologies", In: Hacket et al., The Handbook of Science and Technology Studies, Cambridge, MIT Press, pp. 949-978.

Bowker G. (2005), Memory Practices in the Sciences, Cambridge, MIT Press.

Callon M., Rabeharisoa V. (2008), “The Growing Engagement of Emergent Concerned Groups in Political and Economic Life: Lessons from the French Association of Neuromuscular Disease Patients", Science, Technology, \& Human Values, 33(2), pp. 230-261.

Cardon D., Heurtin J.-P., Martin O., Pharabod A.-S., Rozier S. (1999), « Les formats de la générosité. Trois explorations du Téléthon », Réseaux, 95(17), pp. 15-105.

Carricaburu D. (1993), «L'Association française des hémophiles face au danger de contamination par le virus du sida : stratégie de normalisation de la maladie et définition collective du risque », Sciences Sociales et Santé, vol. 11, n 3-4. p. 55-81.

Dodier N. (2003), Leçons politiques de l'épidémie de sida, Paris, Editions de l'EHESS.

Epstein S. (1995), "The construction of lay expertise : AIDS activism and the forging of credibility in the reform of clinical trials", Science, Technology, \& Human Values, vol. $20, n^{\circ} 4$, pp. $408-437$.

Epstein S. (2008), "Patient groups and health movements", In: Hackett et al., The Handbook of Science and Technology Studies, Cambridge, MIT Press, pp. 499-540.

Fox N. J., Ward K. J., O’Rourke A. J. (2005), “The expert patient: Empowerment or medical dominance? The case of weight loss, pharmaceutical drugs and the internet", Social Science \& Medicine, 30, pp. 1299-1309.

Hardey M. (1999), "Doctor in the house: The internet as a source of lay health knowledge and the challenge to expertise", Sociology of Health \& Illness, 21, pp. 820-35.

Huyard, C. (2007), Rendre le rare commun. Expériences de maladies rares et construction d'une action collective, thèse de sociologie de l'EHESS, Paris.

Huyard C. (2009), «How did uncommon disorders become 'rare diseases'? History of a boundary object », Sociology of Health \& Illness, 31(4), pp. 463-477.

Paterson F., Barral C. (1994), «L'Association française contre les myopathies : trajectoire d'une association d'usagers et construction associative d'une maladie », Sciences Sociales et Santé, vol. 12, n 2, pp. 70-111.

Patterson J. (1987), The dread disease : Cancer and modern American culture, Cambridge, Harvard University Press. 
Peerbaye, A. (2004), La Construction de l'espace génomique en France: la place des dispositifs instrumentaux, thèse de sociologie de l'ENS de Cachan.

Pinell P. (1992), Naissance d'un fléau : histoire de la lutte contre le cancer en France, 18901940, Paris, Métailié.

Rabeharisoa, V., Callon, M. (1999), Le Pouvoir des malades. L'Association française contre les myopathies et la recherche, Presses de l'Ecole des Mines, Paris. 